\title{
Hyperglycemia After Dolutegravir-Based Antiretroviral Therapy
}

\section{Workagegnehu Hailu \\ Tsebaot Tesfaye \\ Abilo Tadesse}

Department of Internal Medicine, University of Gondar, Gondar, North Gondar, Ethiopia
Correspondence: Abilo Tadesse

Tel +25I 91।405I44

Email abilo.tadesse@yahoo.com
Background: Antiretroviral therapies prolong life expectancy and improve the quality of life of HIV-infected patients. Despite the documented benefits of antiretroviral drugs, its use is not without side effects. Here, we report cases of new onset diabetes mellitus after taking a dolutegravir (DTG)-based ART regimen.

Case Presentation: HIV-infected patients who had been on non-nucleoside reverse transcriptase inhibitor (NNRTI)-based ART regimens for more than a decade were shifted to integrase strand transfer inhibitors (dolutegravir)-based ART regimen as recommended by the National Comprehensive HIV Care Guideline. They were diagnosed to have diabetes mellitus with or without diabetic ketoacidosis (DKA) as evidenced by polyuria, polydipsia and fatigue, severe hyperglycemia (plasma glucose level $>250 \mathrm{mg} / \mathrm{dl}$ ) with or without ketonuria (3+) after 1-12 months of DTG-based ART regimen. Two of the patients who presented with DKA were treated with intravenous fluids and regular insulin. NPH insulin was started following recovery from DKA, which later shifted to metformin. One of the patients who presented with severe hyperglycemia without DKA was started with NPH insulin, which later shifted to metformin. Good glycemic control was obtained with metformin, while the DTG-based ART regimen was continued.

Conclusion: Hyperglycemia is a potential and noticed side effect of the DTG-based ART regimen. Baseline and periodic monitoring of plasma glucose might be required in ART regimens containing dolutegravir.

Keywords: INSTIs, dolutegravir, hyperglycemia

\section{Background}

Antiretroviral therapy (ART) restores immune function and reduces HIV-related adverse outcomes. Despite documented benefits of antiretroviral drugs, its use is not without side effects. ${ }^{1,2}$ Protease inhibitors (PIs), and to a lesser extent, nucleoside/ tide reverse transcriptase inhibitors (NRTIs) and non-nucleoside reverse transcriptase inhibitors (NNRTIs) were known to cause deranged glucose and lipid metabolism, leading to hyperglycemia, dyslipidemia and insulin resistance. ${ }^{5,6}$ In addition, insulin resistance might be worsened by immune activation and chronic inflammation due to HIV infection. Recently, there have been a few documented reports on deranged glucose metabolism with integrase strand transfer inhibitors (INSTIs) use. $^{8-17}$ SAILING, SPRING-2, SINGLE and VIKING-3 clinical trials reported the incidence of moderate (plasma glucose, 126-250 mg/dl) and severe hyperglycemia (plasma glucose, $>250 \mathrm{mg} / \mathrm{dl}$ ) were $6-9 \%$ and $1-2 \%$, respectively, after INSTIs therapy. ${ }^{8-11}$ This case series is the first of its kind in Ethiopia to report on new onset diabetes mellitus after dolutegravir-based ART use. 
Table I Laboratory Profiles of HIV Infected Patients Taking Dolutegravir-Based ART Regimen at Initial Admission to Emergency Medical OPD, University of Gondar Hospital, Northwest Ethiopia

\begin{tabular}{|c|c|c|c|c|}
\hline Parameters & Case-I & Case-2 & Case-3 & Ref. \\
\hline \multicolumn{5}{|l|}{ Hemogram } \\
\hline WBC $\left(x 10^{3}\right.$ cells $\left./ \mathrm{mm}^{3}\right)$ & 10.6 & 5.2 & 6.4 & $4.0-11.0$ \\
\hline $\mathrm{Hgb}(\mathrm{gm} / \mathrm{dl})$ & 16.2 & 14.2 & 12.4 & $12-16.5$ \\
\hline Platelets $\left(\left(x \mid 0^{3}\right.\right.$ cells $\left./ \mathrm{mm}^{3}\right)$ & 313 & 158 & 140 & $150-450$ \\
\hline \multicolumn{5}{|l|}{ Urinalysis } \\
\hline Glucosuria & $3+$ & $3+$ & $3+$ & $-v e$ \\
\hline Ketonuria & $3+$ & -ve & $3+$ & $-v e$ \\
\hline Protein & Trace & $1+$ & $-\mathrm{ve}$ & $-v e$ \\
\hline Sediments & - & - & - & $-v e$ \\
\hline \multicolumn{5}{|l|}{ Liver biochemical tests } \\
\hline ALT (IU/L) & - & 23 & - & $0-40$ \\
\hline AST (IU/L) & - & 19 & - & $0-40$ \\
\hline \multicolumn{5}{|l|}{ Renal function tests } \\
\hline Serum $\mathrm{Cr}(\mathrm{mg} / \mathrm{dl})$ & 0.82 & 0.77 & 0.79 & $0.6-1.2$ \\
\hline BUN (mg/dl) & 22 & 27 & 30 & $5-30$ \\
\hline \multicolumn{5}{|l|}{ Electrolytes } \\
\hline Serum $\mathrm{Na}^{+}(\mathrm{meq} / \mathrm{L})$ & 133 & - & - & $135-145$ \\
\hline Serum $K^{+}(\mathrm{meq} / \mathrm{L})$ & 4.4 & 4.2 & 3.9 & $3.5-5.5$ \\
\hline \multicolumn{5}{|l|}{ Blood sugar profile } \\
\hline RBS (mg/dl) & 320 & 378 & 350 & $<180$ \\
\hline HbAlc (\%) & 9.3 & - & 11.4 & $4.0-6.0$ \\
\hline \multicolumn{5}{|l|}{ Lipid profile } \\
\hline Total cholesterol & 160.5 & 180.1 & 227 & $<200$ \\
\hline $\mathrm{HDL}$ & 33.4 & 34.1 & - & $40-60$ \\
\hline LDL & 127.0 & 95.9 & - & $<130$ \\
\hline Triglycerides & 61.6 & 250.2 & - & $<150$ \\
\hline
\end{tabular}

Abbreviations: ALT, alanine aminotransferase; AST, aspartate aminotransferase; BUN, blood urea nitrogen; Cr, creatinine; Hgb, hemoglobin; HbAlc; hemoglobin Alc; HDL, high density lipoprotein; LDL, low density lipoprotein; RBS, random blood sugar; WBC, white blood cells.

\section{Case Presentation}

\section{Case-I}

A 48-year-old male patient was diagnosed with stage II HIV infection 11 years back after presenting with cutaneous fungal infections. He was treated with topical antifungals, and started on ART (AZT-3TC-EFV) and cotrimoxazole preventive therapy (CPT) with a baseline $\mathrm{CD}_{4}{ }^{+}$count 175 cells $/ \mathrm{mm}^{3}$. The patient achieved an undetected viral load after 6 months of ART initiation. The ART regimen was shifted to TDF-3TC-DTG after 10 years as recommended by the National Comprehensive HIV Care Guideline. His fasting blood sugar (FBS) level was $101 \mathrm{mg} / \mathrm{dl}$ at time of the DTG-based ART initiation. He was told to have hypertension on medical follow up and was prescribed amlodipine, $10 \mathrm{mg}$ po daily, and advised on lifestyle modification. He was diagnosed to have diabetic ketoacidosis (DKA) with clinical evidence of polyuria, polydipsia and fatigue, severe hyperglycemia $(\mathrm{RBS}=320-466 \mathrm{mg} / \mathrm{dl}, \mathrm{HbA} 1 \mathrm{c}=9.3 \%)$, and ketonuria $(3$ $+)$ ), which occurred after 7 months of DTG-based ART regimen (Table 1). No documented preceding infections. No personal or family history of diabetes mellitus or dyslipidemia. Serum C-peptide level, anti-insulin antibody or anti-glutamic acid decarboxylase (GAD) antibody were not determined due to limited clinical setup. He was treated with intravenous fluids, regular insulin and $\mathrm{IV} \mathrm{KCl}$, and recovered from DKA after 4 days of admission. NPH insulin 20/10 IU s/c daily was started, which later changed to metformin $850 \mathrm{mg} /$ day. Good glycemic control (FBS=101-130 mg/dl, HbA1c=7.1\%) was obtained with 
metformin, while the DTG-based ART regimen was continued.

\section{Case-2}

A 49-year-old female patient was diagnosed with stage III HIV infection 11 years back after presenting with smear negative pulmonary tuberculosis and oropharyngeal candidiasis with a baseline $\mathrm{CD}_{4}{ }^{+}$count 74 cells $/ \mathrm{mm}^{3}$. She was covered with fluconazole as antifungal therapy. She was treated with anti-tuberculosis treatment (ATT) for 6 months and declared treatment completed. ART (AZT3TC-NVP) and CPT were started two weeks after ATT initiation. She had records of undetected viral load after 2 years of ART initiation. The ART regimen was shifted to TDF-3TC-DTG after 10 years as recommended by the National Comprehensive HIV Care Guideline. She was diagnosed with hypertension and dyslipidemia on followups, and advised on lifestyle modification. She was diagnosed with type 2 diabetes after presenting with polyuria, polydipsia, fatigue and blurred vision, and hyperglycemia $(\mathrm{RBS}=313 \mathrm{~g} / \mathrm{dl})$ which occurred after 1 year of the DTGbased ART regimen (Table 1). No documented preceding infections. No personal or family history of diabetes mellitus. Serum C-peptide level, anti-insulin antibody or antiGAD antibody were not determined due to limited clinical setup. No documented plasma glucose level prior to changing ART regimen. She was started on NPH insulin 24 IU $\mathrm{s} / \mathrm{c}$ daily, which later changed to metformin $1 \mathrm{gm}$ po daily. Good glycemic control (FBS=109-135 mg/dl) was achieved with metformin, while the DTG-based ART regimen was continued.

\section{Case-3}

A 46-year-old female patient was diagnosed with stage I HIV infection after voluntary counseling and testing (VCT) for HIV 13 years back. She was initiated on ART (AZT-3TC-NVP) and CPT 2 years later with a $\mathrm{CD}_{4}{ }^{+}$count 119 cells $/ \mathrm{mm}^{3}$. The ART regimen was shifted to TDF3TC-DTG after 11 years as recommended by the National Comprehensive HIV Care Guideline. She was diagnosed to have DKA with clinical evidence of polyuria, polydipsia and fatigue, severe hyperglycemia $(\mathrm{RBS}=350$ $450 \mathrm{mg} / \mathrm{dl}, \mathrm{HbA} 1 \mathrm{c}=11.4 \%$ ) and ketonuria $(3+)$, which occurred after 1 month of the DTG-based ART regimen (Table 1). No documented preceding infections. No personal or family history of diabetes mellitus or dyslipidemia. Serum C-peptide level, anti-insulin antibody or anti-GAD antibody were not determined due to limited clinical setup.
No documented plasma glucose level prior to changing ART regimen. She was treated with intravenous fluids, regular insulin and $\mathrm{IV} \mathrm{KCl}$, and recovered from diabetic ketoacidosis after 3 days of admission. NPH insulin 10/8 IU s/c daily was initiated, which later changed to metformin $750 \mathrm{mg}$ po daily. Good glycemic control (FBS=106$140 \mathrm{mg} /$ day, $\mathrm{HbA} 1 \mathrm{c}=5.6 \%$ ) was obtained with metformin, while the DTG-based ART regimen was continued.

\section{Discussion}

Antiretroviral drugs have markedly reduced HIV-related morbidity and mortality. It has transformed HIV/AIDS from an inevitably fatal disease to a chronic, manageable illness. Combinations of antiretroviral drugs are required to achieve maximal viral suppression. The ART regimen comprises a combination of three drugs in two classes of antiretroviral drugs, ie, 2 NRTIs and 2 PIs, 1 NNRTIs, or 1 INSTIs. ${ }^{1,2}$ Second-generation INSTIs are currently the class of choice in ART regimens due to their high potency, good tolerability, low toxicity and high genetic barrier to resistance. ${ }^{1-3}$ The dolutegravir-based ART regimen was incorporated into the preferred regimen of choice in sub-Saharan Africa, including Ethiopia., INSTIs have been linked to weight gain and neuropsychiatric disorders. ${ }^{7}$ Recently, there is mounting evidence that INSTIs cause deranged glucose metabolism. ${ }^{8-17}$ Severe hyperglycemia (Plasma glucose level $>250 \mathrm{mg} /$ dl) with or without its life threatening acute complications (diabetic ketoacidosis or hyperosmolar hyperglycemia state) was recognized after INSTIs use in SAILING, SPRING-2, SINGLE, and VIKING-3 clinical trials. ${ }^{8-11}$ Similarly, severe $(250-500 \mathrm{mg} / \mathrm{dl})$ or life threatening (>500 mg/dl) hyperglycemia were documented in case reports by Kamal et al, McLaughlin et al, Ntem-Mensah et al, Horikawa et al and Lamonde et al. ${ }^{12-15}$ INSTIs are believed to work by chelating $\mathrm{mg}^{2+}$ to prevent HIV from integrating into host DNA. Chelating $\mathrm{mg}^{2+}$ by INSTIs may lead to disorders of glucose metabolism, because $\mathrm{mg}^{2+}$ also serves as a cofactor in post-receptor insulin action. ${ }^{13-17}$ Two of the patients who presented with DKA were treated with intravenous fluids and regular insulin. NPH insulin was started following recovery from DKA, which later shifted to metformin. One of the patients who presented with severe hyperglycemia without DKA was started with NPH insulin, which later shifted to metformin. Good glycemic control was obtained with metformin, while the DTG-based ART regimen was continued. In conclusion, hyperglycemia is 
a potential and noticed side effect of the DTG-based ART regimen. Baseline and periodic monitoring of plasma glucose might be required in ART regimens containing dolutegravir.

\section{Abbreviations}

ART, antiretroviral therapy; ATT, anti-tuberculosis treatment; AZT, zidovudine; DKA, diabetic ketoacidosis; DNA, deoxyribonucleic acid; DTG, dolutegravir; EFV, efavirenz; GAD, glutamic acid decarboxylase; HIV, human immunodeficiency virus; INSTIs, integrase strand transfer inhibitors; NVP, nevirapine; NRTIS, nucleoside/ tide reverse transcriptase inhibitors; NNRTIs, nonnucleoside reverse transcriptase inhibitors; PIs, protease inhibitors; TBC, tuberculosis; TDF, tenofovir disoproxil fumarate; 3TC, lamivudine; VCT, voluntary counseling and testing.

\section{Ethics Approval and Consent to Participate}

The authors declare that ethics approval was not required for these case reports as we did not use any new procedures or any treatment, which was not approved for clinical use in our institution.

\section{Consent for Publication}

Written informed consent was obtained from patients for publication of this case series and any accompanying laboratory reports. A copy of the written consent is available for review by the Editor-in-Chief of this journal.

\section{Acknowledgments}

We are grateful to the medical personnel, who were caring for the patients.

\section{Author Contributions}

All authors contributed to data analysis, drafting or revising the article, have agreed on the journal to which the article will be submitted, gave final approval of the version to be published, and agree to be accountable for all aspects of the work.

\section{Funding}

There is no funding to report.

\section{Disclosure}

The authors declare that they have no conflicts of interest for this work.

\section{References}

1. WHO Clinical Guidelines. Antiretroviral therapy; 2020. Avaialble from: http://www.who.int. Accessed July 23, 2021.

2. Ethiopia national consolidated guidelines for comprehensive HIV prevention, care and treatment; 2018. Avaialble from: http://www. afro.who.int. Accessed July 23, 2021.

3. Magdalena DI, Carmen CR, Rugina S. Dolutegravir efficacy in HIV infected patients. ARS Medica Tomitana. 2015;1(21):42-51.

4. Phillips AN, Venter F, Havlir D, et al. Risks and benefits of dolutegravir-based antiretroviral drug regimens in Sub-Saharan Africa: a modeling study. Lancet. 2019;6(2):e116-27. doi:10.1016/ S2352-3018(18)30317-5

5. American Diabetes Association. Diabetes care: standards of medical care in diabetes-2020. J Clin Appl Res Educ. 2020;43(suppl-1):S1S212.

6. Thet D, Siritientong T. Antiretroviral therapy-associated metabolic complications. Review of the recent studies. HIV AIDS (Auckl). 2020;12:507-524. doi:10.2147/HIV.S275314

7. Kolakowska A, Maresca AF, Collins IJ, Cailhol J. Updates on adverse effects on HIV integrase inhibitors. Curr Treat Options Infect Dis. 2020;11(4):372-87. doi:10.1007/S40506-019-00203-7

8. Cahn P, Pozniak AL, Mingrone H, et al. Dolutegravir versus raltegravir in antiretroviral-experienced, integrase-inhibitor-naïve adults with HIV: week 48 results from the randomized, double blind, noninferiority SAILING Study. Lancet. 2013;382(9893):700-708. doi:10.1016/S0140-6736(13)61221-0

9. Raffi F, Rachlis A, Stellbrink HJ, et al. Once-daily dolutegravir versus raltegravir in antiretroviral-naive adults with HIV-1 infection: 48 week results from the randomised, double-blind, non-inferiority SPRING-2 study. Lancet. 2013;381(9868):735-743. doi:10.1016/ S0140-6736(12)61853-4

10. Walmsley SL, Antela A, Clumeck N, et al. Dolutegravir plus abacavirlamivudine for the treatment of HIV-1 infection. $N$ Engl $J$ Med. 2013;369(19):1807-1818. doi:10.1056/NEJMoa1215541

11. Castagna A, Maggiolo F, Penco G, et al. Dolutegravir in antiretroviralexperienced patients with raltegravir- and/or elvitegravir-resistant HIV-1: 24-week results of the Phase III VIKING-3 study. $J$ Infect Dis. 2014;210(3):354-362. doi:10.1093/infdis/jiu051

12. Kamal P, Sharma S. SUN-187 dolutegravir causing diabetes. $J$ Endocr Soc. 2019;3(suppl1):SUN-187. doi:10.1210/js.2019-SUN $-187$

13. McLaughlin M, Walsh S, Galvin S. Dolutegravir-induced hyperglycemia in a patient living with HIV. J Antimicrob Chemother. 2018;73 (1):253-260. doi:10.1093/jac/dkx365

14. Ntem-Mensah AD, Millman N, Jakharia N, et al. Acute onset diabetic ketoacidosis/hyperosmolar hyperglycemic state in patients taking integrase strand transferase inhibitors. Available from: http://aca demic.oup.com/ofid/articles/6/supplement-2/s/183/5604152. Accessed July 23, 2021.

15. Horikawa M, Toyoda M, Saito N, et al. Raltegravir-associated diabetic ketoacidosis in a patient with HIV infection: a case report. Tokai J Exp Clin Med. 2018;43(1):19-23.

16. Lamonde M, Atwiine M, Owarwo N, et al. Dolutegravir-associated hyperglycemia in patients with HIV. Lancet. 2020;7(7):E461-E462. doi:10.1016/S2352-3018(20)30042-4

17. Fong PS, Flynn DM, Evans CD, Korthuis PT. Integrase strand transfer inhibitor-associated diabetes: a case report. Int $J$ STD AIDS. 2016;28(6):626-8. doi:10.1177/0956462416675107 


\section{Publish your work in this journal}

The International Medical Case Reports Journal is an international, peer-reviewed open-access journal publishing original case reports from all medical specialties. Previously unpublished medical posters are also accepted relating to any area of clinical or preclinica science. Submissions should not normally exceed 2,000 words or 4 published pages including figures, diagrams and references. The manuscript management system is completely online and includes a very quick and fair peer-review system, which is all easy to use. Visit http://www.dovepress.com/testimonials.php to read real quotes from published authors. 